\title{
Jacobi multipliers and Hojman symmetry
}

\author{
José F. Cariñena and Manuel F. Rañada \\ Departamento de Física Teórica and IUMA, Universidad de Zaragoza. \\ 50009, Zaragoza, Spain \\ e-mail: jfc@unizar.es, mfran@unizar.es
}

June 24, 2021

\begin{abstract}
The geometric intrinsic approach to Hojman symmetry is developed and use is made of the theory of the Jacobi last multipliers to find the corresponding conserved quantity for non divergence-free vector fields. The particular cases of autonomous Lagrangian and Hamiltonian systems are studied as well as the generalization of these results to normalizer vector fields of the dynamics. The nonautonomous cases, where normalizer vector fields play a relevant role, are also developed.
\end{abstract}

MSC: 70G45, 70H03,70H05.

PACS: 02.30.Hq, 02.40.-k, 45.20.Jj, 76M60, 87.10.Ed.

Keywords: Hojman symmetry, Jacobi multipliers 


\section{Introduction}

The first-integrals of a vector field in a $n$-dimensional manifold $M, X=\sum_{i=1}^{n} X^{i}(x) \partial / \partial x^{i}$, play a very relevant role in its integrability, and therefore in that of the corresponding autonomous system of first-order differential equations:

$$
\dot{x}^{i}=X^{i}(x), \quad i=1, \ldots, n .
$$

Moreover, the knowledge of other tensor fields that are invariant under the given vector field furnishes tools for reduction to simpler cases, and the best known example is the case of an invariant symplectic form $\omega$, for which one of the more celebrated related results is that of the Noether theorem, that in this case amounts to say that if a Hamiltonian vector field $X_{F}$ is a symmetry of the Hamiltonian function $H$, then the function $F$ is a constant of the motion for the dynamical Hamiltonian vector field $X_{H}$. The very simple proof is based on the skew-symmetry of the Poisson bracket defined by the symplectic structure, because $X_{H} F=\{F, H\}=-X_{F} H$. The particular case of regular Lagrangians can be considered as a particular case of a Hamiltonian system where, in this case, the symplectic form $\omega_{L}$ and the Hamiltonian function $H$, the energy function, are defined from the Lagrangian $L$. The symmetries of both the symplectic form and the energy function correspond to the invariance of the Lagrangian, may be up to addition of a gauge term. Just to mention another instance, the existence of an invariant volume form for a vector field $X$ in an oriented manifold, which is equivalent to know a Jacobi multiplier for $X$, was used by Jacobi to prove that if $(n-2)$ first integrals are known, the vector field is integrable by quadratures.

Even if the usual way for finding first-integrals is the method suggested by Noether theorem of introducing symmetries, there is an alternative method for finding nonNoether constants of motion that is not based on symmetries but on the existence of alternative compatible geometric structures for the description of the vector field, what leads to the existence of a recursion operator. The case of the Lagrangian formalism for a system with a one-dimensional configuration space was given in [1], while the multidimensional case was given in [2] and the geometric approach in intrinsic terms was given in [3].

The third approach we want to study here is the one started in a coordinate dependent way by Hojman [4] for the case of a divergence-free non-autonomous system of second-order ordinary differential equations and later on extended by GonzálezGascón in [5] for a non divergence-free case, and that it is becoming more and more 
important during the last years for its applications in $f(R)$-gravity and FRW cosmology [6, 17, 8, 9, 10, 11, 12. The geometric approach to autonomous systems provides a method to incorporate holonomic constraints by replacing the systems by vector fields in a differentiable manifold and allows to develop coordinate free formulations of the problem. In this way the geometric theory of reduction and symmetry may be very helpful. Therefore, our aim in this paper is to present a geometric approach generalising the previously known cases and unifying them in a common simple geometric formulation, by showing that they appear as particular cases of a more general theory.

The paper is organized as follows: Section 2 summarises basic results of the differential geometry concerning the concept of divergence of a vector field and a basic relation between the divergences of two vector fields, and we also recall the fundamental concept of Jacobi multiplier introduced by him to integrate by quadratures a vector field when $(n-2)$ functionally independent first-integrals and a Jacobi multiplier are known. Using these concepts we can introduce some mathematical results which can be used to obtain a first-integral of the vector field $X$ in case that an infinitesimal symmetry $Y$ of $X$ or a normalizer vector field are known. After this geometric presentation we particularise in Section 3 to the cases of autonomous Lagrangian and Hamiltonian systems, while nonautonomous systems of first-order differential equations are analysed in Section 4, and in particular the Hamilton systems. Section 5 is devoted to nonautonomous systems of second-order differential equations with a special emphasis on those admitting a regular Lagrangian formulation. Many results on constants of motion scattered by the physics literature arise here as particular cases of this more general unifying presentation.

\section{First integrals derived from infinitesimal sym- metries of vector fields}

Different relations among vector fields and their divergences can be used to establish first integrals and integral invariants for vector fields in a manifold $M$. Given a vector field $X \in \mathfrak{X}(M)$ in an oriented manifold $(M, \Omega)$, we denote $\operatorname{div}(X)$ the function defined by $\mathcal{L}_{X} \Omega=\operatorname{div}(X) \Omega$. In the particular case of a choice of local coordinates $\left(x^{1}, \ldots, x^{n}\right)$ such that $\Omega=d x^{1} \wedge \cdots \wedge d x^{n}$, if the local expression of $X$ is $X=$ 
$\sum_{i=1}^{n} X^{i} \frac{\partial}{\partial x^{i}}$, then the expression of $\operatorname{div}(X)$ is the usual one:

$$
\operatorname{div}(X)=\sum_{i=1}^{n} \frac{\partial X^{i}}{\partial x^{i}}
$$

Note that for each function $f \in C^{\infty}(M)$, from $\mathcal{L}_{f X} \Omega=\mathcal{L}_{X}(f \Omega)=(X(f)+$ $f \operatorname{div}(X)) \Omega$ we have that $\operatorname{div}(f X)=X(f)+f \operatorname{div}(X)$.

There is a geometric relation playing a fundamental rôle: If $X, Y$ is an arbitrary pair of vector fields in an oriented manifold $(M, \Omega)$, then

$$
\mathcal{L}_{X}(\operatorname{div}(Y))-\mathcal{L}_{Y}(\operatorname{div}(X))=\operatorname{div}([X, Y]),
$$

because as the Lie derivatives of a $p$-form $\alpha$ satisfy the relation

$$
\mathcal{L}_{X}\left(\mathcal{L}_{Y} \alpha\right)-\mathcal{L}_{Y}\left(\mathcal{L}_{X} \alpha\right)=\mathcal{L}_{[X, Y]} \alpha
$$

in the particular case $\alpha=\Omega$, we have

$$
\mathcal{L}_{X}\left(\mathcal{L}_{Y} \Omega\right)-\mathcal{L}_{Y}\left(\mathcal{L}_{X} \Omega\right)=\mathcal{L}_{[X, Y]} \Omega,
$$

and from the difference of

$$
\mathcal{L}_{X}\left(\mathcal{L}_{Y} \Omega\right)=\mathcal{L}_{X}(\operatorname{div}(Y) \Omega)=\mathcal{L}_{X}(\operatorname{div}(Y)) \Omega+\operatorname{div}(Y) \operatorname{div}(X) \Omega
$$

and the corresponding relation

$$
\mathcal{L}_{Y}\left(\mathcal{L}_{X} \Omega\right)=\mathcal{L}_{Y}(\operatorname{div}(X) \Omega)=\mathcal{L}_{Y}(\operatorname{div}(X)) \Omega+\operatorname{div}(X) \operatorname{div}(Y) \Omega
$$

we obtain (2.1).

The following theorem makes use of this geometric property and generalises some previous results which had been obtained in [4, 13, by making use of a coordinate dependent approach:

Theorem 1 Let $X$ be a divergence-free vector field and $Y$ an infinitesimal symmetry of $X$. Then $\operatorname{div}(Y)$ is a constant of the motion for $X$.

Proof.- Let us apply the relation (2.1) to a divergence-free vector field $X$. Then if the vector field $Y \in \mathfrak{X}(M)$ is a symmetry of $X$, it satisfies $[Y, X]=0$ and therefore we obtain that $\mathcal{L}_{X}(\operatorname{div}(Y))=0$. 
Next, in the following two theorems, we present two generalizations of this result. First, we delete the condition for $X$ to be a divergence-free vector field and second, the condition of $Y$ being an exact symmetry is substituted for the weaker condition of symmetry of the one-dimensional distribution generated by $X$. So, if the condition for $X$ to be a divergence-free vector field is eliminated we can use the Jacobi multiplier theory and then we have a more general result obtained in [5, 14. Recall first that a positive function $R \in C^{\infty}(M)$ is a Jacobi multiplier for $X \in \mathfrak{X}(M)$ in the oriented manifold $(M, \Omega)$ if the vector field $R X$ is such that $\mathcal{L}_{R X} \Omega=0$, or equivalently, as $\mathcal{L}_{R X}(\Omega)=d\left(i(R X) \Omega=d(i(X)(R \Omega))=\mathcal{L}_{X}(R \Omega)\right.$, if $\mathcal{L}_{X}(R \Omega)=0$. Note that

$$
\mathcal{L}_{X}(R \Omega)=\mathcal{L}_{X}(R) \Omega+R \mathcal{L}_{X} \Omega=\left(\mathcal{L}_{X}(R)+R \operatorname{div}(X)\right) \Omega,
$$

and therefore the condition for $R$ to be a Jacobi multiplier (see [15] and references therein for more details) becomes

$$
\mathcal{L}_{X}(R)+R \operatorname{div}(X)=0 \Longleftrightarrow \operatorname{div}(X)+\mathcal{L}_{X}(\log R)=0 .
$$

Remark that from the above mentioned property $\mathcal{L}_{X}(R \Omega)=\mathcal{L}_{R X} \Omega$, we see that $R$ is a Jacobi multiplier for $X$ if and only if the vector field $X$ is divergence-free with respect to $R \Omega$, i.e., we see the equivalence of searching for Jacobi multipliers on one side and the more geometric concept of invariant volume-forms on the other: a volume form $\Omega^{\prime}=R \Omega$ is $X$-invariant, i.e. $\mathcal{L}_{X} \Omega^{\prime}=0$, if and only if $R$ is a Jacobi multiplier for the vector field $X$ in the oriented manifold $(M, \Omega)$. Moreover, this property also shows that $R$ is a Jacobi multiplier for $X$ in the oriented manifold $(M, \Omega)$ iff and only if $f R$ is a Jacobi multiplier for $X$ in the oriented manifold $\left(M, f^{-1} \Omega\right)$, for each positive function $f$.

Theorem 2 If the function $R$ is a Jacobi multiplier for the vector field $X$ and the vector field $Y$ is an infinitesimal symmetry of $X$, i.e. $[X, Y]=0$, then the function

$$
I=\operatorname{div}(Y)+Y(\log R)
$$

is a constant of the motion for $X$.

Proof.- If $[X, Y]=0$, then according to the relation (2.1) for such a pair $X, Y$, of vector fields we have that $\mathcal{L}_{X} \operatorname{div}(Y)=\mathcal{L}_{Y} \operatorname{div}(X)$. If $R$ is a Jacobi multiplier for the vector field $X$, then (2.4) shows that $\operatorname{div}(X)=-\mathcal{L}_{X}(\log R)$, and consequently in this case the equation (2.1) can be rewritten as

$$
\mathcal{L}_{X} \operatorname{div}(Y)=-\mathcal{L}_{Y}\left(\mathcal{L}_{X}(\log R)\right)=-\mathcal{L}_{X}\left(\mathcal{L}_{Y}(\log R)\right) .
$$


Therefore, $\mathcal{L}_{X} I=0$.

Another important case is when the vector field $Y$ is not a symmetry of the vector field $X$ but only of the one-dimensional distribution spanned by $X$, i.e. there exists a function $h \in C^{\infty}(M)$ such that $[Y, X]=h X$. In this case the results of the previous theorems can be generalised to:

Theorem 3 i) If the vector field $X$ is divergence-free and the vector field $Y$ is an infinitesimal symmetry of the one-dimensional distribution generated by $X$, i.e. $[Y, X]=h X$, then the function $\operatorname{div}(Y)+h$ is a constant of the motion for $X$.

ii) If the function $R$ is a Jacobi multiplier for the vector field $X$ and the vector field $Y$ is an infinitesimal symmetry of the one-dimensional distribution generated by $X$, i.e. i.e. there exists a function $h \in C^{\infty}(M)$ such that $[Y, X]=h X$, then the function

$$
I=\operatorname{div}(Y)+Y(\log R)+h
$$

is a constant of the motion for $X$.

Proof.- i) If $\operatorname{div}(X)=0$, then $\mathcal{L}_{X} \Omega=0$, and hence, using (2.1),

$$
\mathcal{L}_{X}\left(\mathcal{L}_{Y} \Omega\right)=\mathcal{L}_{Y}\left(\mathcal{L}_{X} \Omega\right)+\mathcal{L}_{-h X} \Omega=-\mathcal{L}_{X}(h \Omega)=-X(h) \Omega,
$$

and therefore, from

$$
\mathcal{L}_{X}\left(\mathcal{L}_{Y} \Omega\right)=\mathcal{L}_{X}(\operatorname{div}(Y) \Omega)=\mathcal{L}_{X}(\operatorname{div}(Y)) \Omega
$$

we obtain:

$$
\mathcal{L}_{X}(\operatorname{div}(Y)+h)=0 .
$$

ii) Using the relation (2.1) for the vector fields $X$ and $Y$, and the Jacobi multiplier condition $\operatorname{div}(X)+X(\log R)=0$, together with $[Y, X]=h X$, we find that

$$
\mathcal{L}_{X} \operatorname{div}(Y)-\mathcal{L}_{Y}\left(-\mathcal{L}_{X}(\log R)\right)=-\operatorname{div}(h X)=-X(h)-h \operatorname{div}(X),
$$

and as the commutator $\left[\mathcal{L}_{X}, \mathcal{L}_{Y}\right]$ is $\mathcal{L}_{-h Y}$, the preceding relation can be rewritten as

$$
\mathcal{L}_{X}(\operatorname{div}(Y)+Y(\log R))+\mathcal{L}_{h X}(\log R)=-X(h)+h X(\log R)
$$

and simplifying terms,

$$
\mathcal{L}_{X}(\operatorname{div}(Y)+Y(\log R)+h)=0
$$


Given a vector field $X \in \mathfrak{X}(M)$ the vector fields $Y \in \mathfrak{X}(M)$ that are symmetries of the distribution generated by $X$ are sometimes called normalizers of $X$.

With the particular choice of local coordinates $\left(x^{1}, \ldots, x^{n}\right)$ such that $\Omega=d x^{1} \wedge$ $\cdots \wedge d x^{n}$, if the local expressions of the vector fields $X$ and $Y$ are

$$
X=\sum_{i=1}^{n} X^{i} \frac{\partial}{\partial x^{i}}, \quad Y=\sum_{i=1}^{n} Y^{i} \frac{\partial}{\partial x^{i}},
$$

the coordinate expression of constant of motion (2.6) is

$$
I=\sum_{i=1}^{n} \frac{\partial Y^{i}}{\partial x^{i}}+\sum_{i=1}^{n} Y^{i} \frac{\partial(\log R)}{\partial x^{i}}+h,
$$

and the condition $[Y, X]=h X$ means that

$$
Y\left(X^{i}\right)-X\left(Y^{i}\right)=h X^{i} .
$$

\section{Hojman symmetry in the Lagrangian and Hamil- tonian formalism}

We can extend the preceding results to systems of second-order differential equations in normal form,

$$
\ddot{x}^{i}=F^{i}(x, \dot{x}), \quad i=1, \ldots, n,
$$

as done in [5, 14, and the particular case in which this system is equivalent to the set of Euler-Lagrange equations of a regular Lagrangian is of a special interest.

Remark that such a system of $n$ second-order differential equation can be associated with a system of $2 n$ first-order differential equations

$$
\left\{\begin{array}{l}
\dot{x}^{i}=v^{i} \\
\dot{v}^{i}=F^{i}(x, v)
\end{array}, \quad i=1, \ldots, n,\right.
$$

that as a particular case of (1.1) has associated the vector field

$$
\Gamma=\sum_{i=1}^{n}\left(v^{i} \frac{\partial}{\partial x^{i}}+F^{i}(x, v) \frac{\partial}{\partial v^{i}}\right) .
$$


When the configuration space $Q$ of the system is a $n$-dimensional Euclidean space the Euclidean coordinates $\left(x^{1}, \ldots, x^{n}\right)$ induce global coordinates in its tangent bundle, denoted $\left(x^{1}, \ldots, x^{n}, v^{1}, \ldots, v^{n}\right)$ and there is a natural volume element (tangent and cotangent bundles can be identified by the Euclidean metric) given by $\Omega=d x^{1} \wedge \cdots \wedge$ $d x^{n} \wedge d v^{1} \wedge \cdots \wedge d v^{n}$. If the dynamical vector field (3.3) is determined by the Lagrangian $L$, i.e. $i(\Gamma) \omega_{L}=d E_{L}$ (see e.g [16]), we know (see e.g. [15] and references therein) that a particular Jacobi multiplier for given by the determinant of the Hessian matrix in the velocities, with elements $W_{i j}=\partial^{2} L / \partial v^{i} \partial v^{j}$. Therefore, the constant of motion obtained in [14] is just a particular case of the expression (2.5) for $R$ equal to the determinant of the Hessian matrix $W$. In the more general case of $Q$ a $n$-dimensional manifold, a local chart of coordinates $\left(x^{1}, \ldots, x^{n}\right)$ for $Q$ induces a local chart of coordinates in its tangent bundle, denoted $\left(x^{1}, \ldots, x^{n}, v^{1}, \ldots, v^{n}\right)$, and an associated volume element in such a chart given by $\Omega=d x^{1} \wedge \cdots \wedge d x^{n} \wedge d v^{1} \wedge \cdots \wedge d v^{n}$. If the dynamical vector field $\Gamma$ given in such coordinates by (3.3) is determined by the Lagrangian $L$, i.e. $i(\Gamma) \omega_{L}=d E_{L}$, which implies $\mathcal{L}_{\Gamma} \omega_{L}=0$ (see e.g [16]), we know (see e.g. [15] and references therein) that a particular Jacobi multiplier for $\Gamma$ with respect to the volume form $\Omega$ is given by the determinant of the Hessian matrix $W$ in the velocities, with elements $W_{i j}=\partial^{2} L / \partial v^{i} \partial v^{j}$. Actually, from $\mathcal{L}_{\Gamma} \omega_{L}=0$, we see that $\left(\omega_{L}\right)^{\wedge n}$ is an invariant volume under $\Gamma$, and the proportionality factor of $\left(\omega_{L}\right)^{\wedge n}$ and $\Omega$ is det $W$. Therefore, the constant of motion obtained in [14] is just a particular case of the expression (2.5) for $R$ equal to the determinant of the Hessian matrix $W$.

Let us now consider the coordinate expression of the vector field $Y$ defined in $T Q$ with local coordinates $\left(x^{i}, v^{i}\right)$

$$
Y=\sum_{i=1}^{n}\left(Y^{i}(x, v) \frac{\partial}{\partial x^{i}}+\bar{Y}^{i}(x, v) \frac{\partial}{\partial v^{i}}\right),
$$

where the fuctions $\bar{Y}^{i}$ are given by $\bar{Y}^{i}=\Gamma\left(Y^{i}\right)$, because of the symmetry condition $[\Gamma, Y]=0$. Then the coordinate expressions of the constants of motion $I$ given in Theorem 1 and in (2.5) of Theorem 2 for the vector field $\Gamma$ are given, respectively, by

$$
I=\sum_{i=1}^{n}\left(\frac{\partial Y^{i}}{\partial x^{i}}+\frac{\partial \bar{Y}^{i}}{\partial v^{i}}\right)
$$

and

$$
I=\sum_{i=1}^{n}\left(\frac{\partial Y^{i}}{\partial x^{i}}+\frac{\partial \bar{Y}^{i}}{\partial v^{i}}\right)+\frac{1}{R} \sum_{i=1}^{n}\left(Y^{i} \frac{\partial R}{\partial x^{i}}+\bar{Y}^{i} \frac{\partial R}{\partial v^{i}}\right),
$$

These expressions coincide with those of [6, 7]. 
Similarly, let us now consider the time evolution in phase space given in local coordinates $\left(q^{1}, \cdots, q^{n}, p_{1}, \cdots, p_{n}\right)$ of the cotangent bundle $\pi: T^{*} Q \rightarrow Q$ induced from a chart $\left(q^{1}, \ldots, q^{n}\right)$ in the base $Q$ by

$$
\left\{\begin{array}{l}
\dot{q}^{i}=Q^{i}(q, p) \\
\dot{p}_{i}=P_{i}(q, p)
\end{array}\right.
$$

whose solutions correspond to the integral curves of the vector field

$$
X=\sum_{i=1}^{n}\left(Q^{i}(q, p) \frac{\partial}{\partial q^{i}}+P_{i}(q, p) \frac{\partial}{\partial p_{i}}\right) .
$$

and let $\Omega$ be the volume form induced from exterior product of its natural symplectic structure, $\Omega=d q^{1} \wedge \cdots d q^{n} \wedge d p_{1} \wedge \ldots d p_{n}$. Recall that the cotangent bundle is the local prototype of an exact symplectic manifold. There is a canonical 1-form $\theta \in \bigwedge^{1}\left(T^{*} Q\right)$ defined by $\theta_{\alpha}=\pi_{\alpha}^{*} \alpha$, with local expression $\theta=\sum_{i=1}^{n} p_{i} d q^{i}$. Then $\omega=-d \theta$ is a symplectic form on $T^{*} Q$ and the local coordinates are Darboux coordinates.

Then if $R$ is a Jacobi multiplier for $X$, satisfying therefore the equation (2.4),

$$
\sum_{i=1}^{n}\left(\frac{\partial Q^{i}}{\partial q^{i}}+\frac{\partial P_{i}}{\partial p_{i}}\right)+X(\log R)=0
$$

and the vector field $Y$, given in these local coordinates by

$$
Y=\sum_{i=1}^{n}\left(\xi^{i}(q, p) \frac{\partial}{\partial q^{i}}+\eta_{i}(q, p) \frac{\partial}{\partial p_{i}}\right),
$$

is a symmetry of the vector field $X$, the constant of motion (2.5) for $X$ is in this case

$$
I=\sum_{i=1}^{n}\left(\frac{\partial \xi^{i}}{\partial q^{i}}+\frac{\partial \eta_{i}}{\partial p_{i}}+\xi^{i} \frac{\partial(\log R)}{\partial q^{i}}+\eta_{i} \frac{\partial(\log R)}{\partial p_{i}}\right),
$$

as found in [17]. Of course, if $(M, \omega)$ is a symplectic manifold and $X$ is a Hamiltonian vector field and we consider the volume form in $M$ obtained by exterior products of the symplectic form, as $X$ is divergence-free, the multiplier $R$ is just a constant.

Finally, if the vector field $Y$ is a normalizer for $X$, i.e., there exists a fuction $h \in C^{\infty}(M)$ such that $[Y, X]=h X$, then the constant of motion corresponding to that of Theorem 3 is

$$
I=\sum_{i=1}^{n}\left(\frac{\partial \xi^{i}}{\partial q^{i}}+\frac{\partial \eta_{i}}{\partial p_{i}}+\xi^{i} \frac{\partial(\log R)}{\partial q^{i}}+\eta_{i} \frac{\partial(\log R)}{\partial p_{i}}\right)+h .
$$




\section{Hojman symmetry for nonautononous systems of first-order differential equations}

In the geometric approach to non-autonomous systems of first-order differential equations,

$$
\dot{x}^{i}=X^{i}(t, x), \quad i=1, \ldots, n,
$$

time is a new coordinate and then we can change the parameter of the solution curves. This amounts to consider the curves not as parametrized curves but simply as one-dimensional submanifolds and to consider as equivalent two curves that are related by a reparametrization. Therefore we associate with such a non-autonomous system a one-dimensional, and therefore integrable, distribution, generated either by a vector field $X$ in $\mathbb{R} \times M$, or by any other proportional one, $f X$, with $f$ a nonvanishing function, in such a way that its one-dimensional integral manifolds are the equivalence class of integral curves of a representative of the vector field. We can choose, for instance

$$
X=\frac{\partial}{\partial t}+\sum_{i=1}^{n} X^{i}(t, x) \frac{\partial}{\partial x^{i}}
$$

if for a vector field of the distribution we have $\langle d t, X\rangle \neq 0$.

The concept of infinitesimal symmetry must be changed and we say that the vector field $Y \in \mathfrak{X}(\mathbb{R} \times M)$ is a symmetry when the image under its local flow of an integral curve of $X$ is, up to a reparametrization, an integral curve of $X$. This condition means that there exists a function $\lambda \in C^{\infty}(\mathbb{R} \times M)$ such that $[Y, X]=\lambda X$, or in other terms, that the one-dimensional distribution generated by $X$ is invariant under the flow of the vector field $Y$. Note that if the coordinate expression of the vector field $Y$ is

$$
Y=Y^{0}(t, x) \frac{\partial}{\partial t}+\sum_{i=1}^{n} Y^{i}(t, x) \frac{\partial}{\partial x^{i}},
$$

then the condition $[Y, X]=\lambda X$ implies that $\lambda=-X\left(Y^{0}\right)$, as a consequence of the explicit form (4.2) of the representative vector field $X$. In this non-autonomous case, if either $M$ is an Euclidean space or choosing a local chart in $M$, we have the associated the volume form in $\mathbb{R} \times M$ given by $\Omega=d t \wedge d x^{1} \wedge \cdots \wedge d x^{n}$ and then $\operatorname{div}(X)$ and $\operatorname{div}(Y)$ take the form in such coordinates

$$
\operatorname{div}(X)=\sum_{i=1}^{n} \frac{\partial X^{i}}{\partial x^{i}}, \quad \operatorname{div}(Y)=\frac{\partial Y^{0}}{\partial t}+\sum_{i=1}^{n} \frac{\partial Y^{i}}{\partial x^{i}}
$$


The results corresponding to Theorem 1, having in mind the new concept of symmetry is, according to $i$ ) of Theorem 3 .

Theorem 4 i) If the vector field $X$ is divergence-free and the vector field $Y$ is an infinitesimal symmetry of the one-dimensional distribution generated by $X$, then the function $\operatorname{div}(Y)-X\left(Y^{0}\right)$ is a constant of the motion for $X$.

ii) If the function $R$ is a Jacobi multiplier for the vector field $X$ and the vector field $Y$ is an infinitesimal symmetry of the one-dimensional distribution generated by $X$, then the function

$$
I=\operatorname{div}(Y)+Y(\log R)-X\left(Y^{0}\right)
$$

is a constant of the motion for $X$.

In the particular case of $M=T^{*} Q$, if we choose a chart for $\mathbb{R} \times T^{*} Q$ induced from a local chart for $Q$ and the local expression of the vector field $X$ is

$$
X=\frac{\partial}{\partial t}+\sum_{i=1}^{n}\left(Q^{i}(t, q, p) \frac{\partial}{\partial q^{i}}+P_{i}(t, q, p) \frac{\partial}{\partial p_{i}}\right)
$$

which corresponds to a non-autonomous system as (3.7) but where now the functions $Q$ and $P$ also depend on $t$, if the coordinate expression of a vector field $Y$ on $\mathbb{R} \times T^{*} Q$ is

$$
Y=\sigma \frac{\partial}{\partial t}+\sum_{i=1}^{n}\left(\xi^{i} \frac{\partial}{\partial q^{i}}+\eta_{i} \frac{\partial}{\partial p_{i}}\right)
$$

then, as

$$
[Y, X]=-\left(X(\sigma) \frac{\partial}{\partial t}+\sum_{i=1}^{n}\left(\left(Y\left(Q^{i}\right)-X\left(\xi^{i}\right)\right) \frac{\partial}{\partial q^{i}}+\left(Y\left(P_{i}\right)-X\left(\eta_{i}\right)\right) \frac{\partial}{\partial p_{i}}\right)\right.
$$

the conditions for $Y$ to be a normalizer of $X,[Y, X]=h X$, are $h=-X(\sigma)$ and $Y\left(Q^{i}\right)-X\left(\xi^{i}\right)=-X(\sigma) Q^{i}$ together with $Y\left(P_{i}\right)-X\left(\eta^{i}\right)=-X(\sigma) P_{i}$, and in thus case the associated constant of motion, for each Jacobi multiplier $R$ for $X$ with respect to the volume form $d t \wedge d q^{1} \wedge \cdots \wedge d q^{n} \wedge d p_{1} \wedge \cdots d p_{n}$ is

$$
I=\frac{1}{R} \frac{\partial(R \sigma)}{\partial t}+\frac{1}{R} \sum_{i=1}^{n}\left(\frac{\partial\left(R \xi^{i}\right)}{\partial q^{i}}+\frac{\partial\left(R \eta_{i}\right)}{\partial p_{i}}\right)-X(\sigma) .
$$

what shows that the results indicated in [18] are but particular cases of the more general theory developed here. 


\section{$5 \quad$ Hojman symmetry for nonautononous systems of second-order differential equations}

A non-autonomous systems of second-order differential equations,

$$
\frac{d^{2} x^{i}}{d t^{2}}=F^{i}(t, x, \dot{x}), \quad i=1, \ldots, n
$$

has an associated system of $(2 n+1)$ first-order differential equations

$$
\left\{\begin{array}{l}
\frac{d t}{d s}=1 \\
\frac{d x^{i}}{d s}=v^{i} \\
\frac{d v^{i}}{d s}=F^{i}(t, x, v)
\end{array} \quad i=1, \ldots, n\right.
$$

which is a particular case of (4.1) and it is therefore geometrically described by a onedimensional distribution $\mathcal{D}_{\Gamma}$ generated by a vector field $\Gamma \in \mathfrak{X}\left(\mathbb{R} \times T \mathbb{R}^{n}\right)$, according to (4.2), given by

$$
\Gamma=\frac{\partial}{\partial t}+\sum_{i=1}^{n}\left(v^{i} \frac{\partial}{\partial x^{i}}+F^{i}(t, x, v) \frac{\partial}{\partial v^{i}}\right) .
$$

Moreover, in the geometric approach $\mathbb{R}^{n}$ is replaced by a $n$-dimensional manifold $Q$ and (5.3) is the local expression of a vector field in the so-called evolution space $E=\mathbb{R} \times T Q$. Remark that $\pi_{1}: \mathbb{R} \times Q \rightarrow \mathbb{R}$ defines a trivial bundle and the jet-bundle of its sections is $J^{1}\left(\pi_{1}\right)=\mathbb{R} \times T Q$.

This vector field $\Gamma$ can be characterized by the vanishing of the $2 n 1$-forms in $\mathbb{R} \times T Q$

$$
\left\{\begin{aligned}
\theta^{i} & =d x^{i}-v^{i} d t, \\
\Psi^{i} & =d v^{i}-F^{i}(t, x, v) d t,
\end{aligned}\right.
$$

together with the condition $\langle d t, \Gamma\rangle=1$.

The first $n 1$-forms $\theta^{i}$ are called contact 1 -forms. Any other representative of the distribution $\mathcal{D}_{\Gamma}$ will be of the form

$$
\bar{\Gamma}=\lambda(t, x, v) \Gamma=\lambda(t, x, v)\left(\frac{\partial}{\partial t}+\sum_{i=1}^{n}\left(v^{i} \frac{\partial}{\partial x^{i}}+F^{i}(t, x, v) \frac{\partial}{\partial v^{i}}\right)\right),
$$

with $\lambda(t, x, v)$ a nonvanishing function. Vector fields of type (5.5) are called SODE vector fields while those of type (5.3) are said to be Newtonian SODE (hereafter shortened as NSODE) vector fields. 
We follow notation of [19] and we therefore refer to such a paper for definitions. More details can be found in [20, 21]. An important point is that the set of 1-forms

$$
\left\{d t, \theta^{i}=d x^{i}-v^{i} d t, \Psi^{i}=d v^{i}-F^{i}(t, x, v) d t\right\}
$$

is a local basis of 1 -forms in $\mathbb{R} \times T Q$ which is the dual basis of vector fields in $\mathfrak{X}(\mathbb{R} \times T Q)$, given by

$$
\left\{\Gamma, \frac{\partial}{\partial x^{i}}, \frac{\partial}{\partial v^{i}}\right\}
$$

Note that while the contact forms only depend on the evolution space, the $n 1$-forms $\Psi^{i}$ depend also on the selected NSODE vector field $\Gamma$. Particular examples for this case of second order differential equations were studied in [4, 5, 13].

We only want to remark here that the Lagrangian formalism is defined for a function $L \in C^{\infty}(\mathbb{R} \times T Q)$ by means of a $(1,1)$-tensor in the evolution space $E$, called vertical endomorphism, whose local coordinate expression is

$$
S=\sum_{i=1}^{n} \frac{\partial}{\partial v^{i}} \otimes \theta^{i}=\sum_{i=1}^{n} \frac{\partial}{\partial v^{i}} \otimes\left(d x^{i}-v^{i} d t\right)
$$

This may be used to define the 1 -form $\Theta_{L}=L d t+d L \circ S$ in $E$,

$$
\Theta_{L}=L d t+\langle d L \circ S, \Gamma\rangle d t+\sum_{i=1}^{n}\left\langle d L \circ S, \frac{\partial}{\partial x^{i}}\right\rangle \theta^{i}+\sum_{i=1}^{n}\left\langle d L \circ S, \frac{\partial}{\partial v^{i}}\right\rangle \Psi^{i}
$$

and as $S(\Gamma)=0$ and $S\left(\partial / \partial v^{i}\right)=0, \forall i=1, \ldots, n$, we find the coordinate expression of $\Theta_{L}$,

$$
\Theta_{L}=L d t+\sum_{i=1}^{n} \frac{\partial L}{\partial v^{i}} \theta^{i}
$$

The exterior differential $d \Theta_{L}$ is used to define the 2 -form $\Omega_{L}=-d \Theta_{L}$, called Cartan 2-form. When $\Omega_{L}$ is of rank $2 n$ the Lagrange function is called regular. In this case, there is only one vector field $\Gamma$ such that $i(\Gamma) \Omega_{L}=0$ and $\langle d t, \Gamma\rangle=1$, which is the dynamical vector field defined by the regular function $L$.

The coordinate expression of the Cartan 2-form is:

$$
\Omega_{L}=-d \Theta_{L}=\sum_{i, j=1}^{n}\left(-A_{i j} \theta^{i} \wedge \theta^{j}+W_{i j} \theta^{i} \wedge \Psi^{j}\right)
$$


with the functions $A_{i j}$ and $W_{i j}$ being given by

$$
A_{i j}=\frac{\partial^{2} L}{\partial x^{i} \partial v^{j}}, \quad W_{i j}=\frac{\partial^{2} L}{\partial v^{i} \partial v^{j}}, \quad i, j=1, \ldots, n .
$$

We recall that the dynamical vector field $\Gamma$ satisfies the two conditions $i(\Gamma) \theta^{i}=0$ and $i(\Gamma) \Psi^{j}=0$ and, because of this, it satisfies also the equation $i(\Gamma) \Omega_{L}=0$. Furthermore, $\left(\Omega_{L}\right)^{\wedge n}$ is a real multiple of $\left(\operatorname{det} W_{i j}\right) \theta^{1} \wedge \ldots \wedge \theta^{n} \wedge \Psi^{1} \wedge \ldots \wedge \Psi^{n}$. Note also that as $d t \wedge \theta^{i}=d t \wedge d x^{i}$ and $d t \wedge \Psi^{i}=d t \wedge d v^{i}$, we have that $d t \wedge\left(\Omega_{L}\right)^{\wedge n}=\left(\operatorname{det} W_{i j}\right) \bar{\Omega}$ where $\bar{\Omega}$ denotes the volume form in the evolution space $\bar{\Omega}=d t \wedge d x^{1} \wedge \cdots \wedge d x^{n} \wedge$ $d v^{1} \wedge \cdots \wedge d v^{n}$. But as $\mathcal{L}_{\Gamma} \Omega_{L}=d\left(i(\Gamma) \Omega_{L}\right)=0$ and $\mathcal{L}_{\Gamma} d t=0$, we have that the vector field $\Gamma$ is divergence-free with respect to the volume form $d t \wedge\left(\Omega_{L}\right)^{\wedge n}$, Consequently the determinant of the matrix with elements $W_{i j}$ is a Jacobi multiplier with respect to the volume form in the evolution space $\bar{\Omega}=d t \wedge d x^{1} \wedge \cdots \wedge d x^{n} \wedge d v^{1} \wedge \cdots \wedge d v^{n}$.

Therefore, this allows us to find as a particular case the result of [14], because if the vector field with coordinate expression

$$
Y=Y^{0}(t, x, \dot{x}) \frac{\partial}{\partial t}+\sum_{i=1}^{n}\left(Y^{i}(t, x, \dot{x}) \frac{\partial}{\partial x^{i}}+\bar{Y}^{i}(t, x, \dot{x}) \frac{\partial}{\partial v^{i}}\right),
$$

is a symmetry of the vector field $\Gamma$ derived from a Lagrangian $L$, then, according to (2.5) the function

$$
I=\operatorname{div} Y+Y(\log W)
$$

is such that $\Gamma(I)=0$, where in this case the divergence should be computed with respect to the volume form $\Omega$ :

$$
\operatorname{div} Y=\frac{\partial Y^{0}}{\partial t}+\sum_{i=1}^{n}\left(\frac{\partial Y^{i}}{\partial x^{i}}+\frac{\partial \bar{Y}^{i}}{\partial v^{i}}\right)
$$

Having in mind that

$$
[Y, \Gamma]=-\Gamma\left(Y^{0}\right) \frac{\partial}{\partial t}+\sum_{i=1}^{n}\left(\left(\bar{Y}^{i}-\Gamma\left(Y^{i}\right)\right) \frac{\partial}{\partial x^{i}}+\left(Y\left(F^{i}\right)-\Gamma\left(\bar{Y}^{i}\right)\right) \frac{\partial}{\partial v^{i}}\right)
$$

we see that $[Y, \Gamma]=0$ if and only if $\Gamma\left(Y^{0}\right)=0, \bar{Y}^{i}=\Gamma\left(Y^{i}\right)$ and $\Gamma\left(\Gamma\left(Y^{i}\right)\right)=Y\left(F^{i}\right)$. Furthermore, $Y$ is an infinitesimal symmetry of the distribution generated by $\Gamma$, i.e. there exists a function $h \in C^{\infty}(M)$ such that $[Y, \Gamma]=h \Gamma$, if and only if $h=-\Gamma\left(Y^{0}\right)$, $\bar{Y}^{i}=\Gamma\left(Y^{i}\right)+h v^{i}$ and $Y\left(F^{i}\right)=\Gamma\left(\bar{Y}^{i}\right)+h F^{i}$. Therefore we obtain

$$
\bar{Y}^{i}=\Gamma\left(Y^{i}\right)-\Gamma\left(Y^{0}\right) v^{i}
$$


and

$$
Y\left(F^{i}\right)=\Gamma\left(\Gamma\left(Y^{i}\right)\right)-2 \Gamma\left(Y^{0}\right) F^{i}-\Gamma\left(\Gamma\left(Y^{0}\right)\right) v^{i}
$$

The two particular cases $Y^{0} \equiv 0$ and $Y^{i} \equiv 0$ were studied in [22].

Particularly interesting cases are those for which the functions $Y^{0}$ and $Y^{i}$ do not depend on velocities because in this case $\Gamma\left(Y^{0}\right)$ and $\Gamma\left(Y^{i}\right)$ do not depend on the choice of the NSODE vector field $\Gamma$ but only of its NSODE character. On the other side, these vector fields play a relevant role in the geometric formulation of Noether theorem, where the symmetry of the dynamical vector field is analysed in terms of symmetry under this kind of vector fields of the Lagrangian itself. We first recall some basic notions of geometry of the evolution space.

In the study of nonautonomous systems of second-order differential equations the contact 1 -forms $\theta^{i}$ play a relevant role, because they can be used to characterize curves $\gamma$ in $\mathbb{R} \times T Q \rightarrow \mathbb{R}$ that are prolongations of sections of the trivial bundle $\mathbb{R} \times Q$ over $\mathbb{R}$ : In fact, such curves are characterized by $\gamma^{*} \theta^{i}=0$, for $i=1, \ldots, n$.

Accordingly, given a vector field $X \in \mathfrak{X}(\mathbb{R} \times Q)$ there exists one vector field $X^{(1)} \in$ $\mathfrak{X}(\mathbb{R} \times T Q)$ such that it is projectable on $X$ and preserves the contact distribution. If the coordinate expression of $X \in \mathfrak{X}(\mathbb{R} \times Q)$ is

$$
X=X^{0}(t, x) \frac{\partial}{\partial t}+\sum_{i=1}^{n}\left(X^{i}(t, x) \frac{\partial}{\partial x^{i}}\right)
$$

then a vector field $X^{(1)} \in \mathfrak{X}(\mathbb{R} \times T Q)$ projectable on $X$, must be of the form

$$
X^{(1)}=X^{0}(t, x) \frac{\partial}{\partial t}+\sum_{i=1}^{n}\left(X^{i}(t, x) \frac{\partial}{\partial x^{i}}+\bar{X}^{i}(t, x, v) \frac{\partial}{\partial v^{i}}\right),
$$

where, choosing an arbitrary NSODE vector field and the corresponding associated bases of 1-forms and vector fields, as

$$
\mathcal{L}_{X^{(1)}} \theta^{i}=\sum_{j=1}^{n}\left(\frac{\partial X^{i}}{\partial x^{j}}-v^{i} \frac{\partial X^{0}}{\partial x^{j}}\right) \theta^{j}+\left(\Gamma X^{i}-\bar{X}^{i}-v^{i} \Gamma X^{0}\right) d t
$$

this shows that in order to the set of contact 1 -forms be preserved by $X^{(1)}$, it must be of the form

$$
\bar{X}^{i}=\Gamma\left(X^{i}\right)-v^{i} \Gamma\left(X^{0}\right), \quad i=1, \ldots, n .
$$

Note that the last relations are valid for any NSODE $\Gamma$.

Next we consider a particular case that contains some of the results obtained in 22 . 
In the particular case of a vector field $X^{(1)}$ that is a symmetry of the onedimensional distribution generated by a NSODE vector field $\Gamma$ determined by a Lagrangian $L$, as $\operatorname{det} W$ is a Jacobi multiplier for $\Gamma$ the Hojman constant of the motion according to (2.6) is given by

$$
I=2 \sum_{i=1}^{n}\left(\frac{\partial X^{i}}{\partial x^{i}}-v^{i} \frac{\partial X^{0}}{\partial x^{i}}\right)-n \Gamma\left(X^{0}\right)+X^{(1)}(\log (\operatorname{det} W)),
$$

because

$$
\operatorname{div}\left(X^{(1)}\right)=\frac{\partial X^{0}}{\partial t}+\sum_{i=1}^{n}\left(\frac{\partial X^{i}}{\partial x^{i}}+\frac{\partial \bar{X}^{i}}{\partial v^{i}}\right)
$$

and as $X^{0}$ and $X^{i}$ do not depend on the velocities,

$$
\frac{\partial\left(\Gamma\left(X^{0}\right)\right)}{\partial v^{i}}=\frac{\partial X^{0}}{\partial x^{i}}, \quad \frac{\partial\left(\Gamma\left(X^{i}\right)\right)}{\partial v^{i}}=\frac{\partial X^{i}}{\partial x^{i}}
$$

and then,

$$
\operatorname{div}\left(X^{(1)}\right)=\frac{\partial X^{0}}{\partial t}+\sum_{i=1}^{n}\left(\frac{\partial X^{i}}{\partial x^{i}}+\frac{\partial\left(\Gamma\left(X^{i}\right)-v^{i} \Gamma\left(X^{0}\right)\right)}{\partial v^{i}}\right)
$$

and therefore

$$
\operatorname{div}\left(X^{(1)}\right)=\frac{\partial X^{0}}{\partial t}+\sum_{i=1}^{n}\left(2 \frac{\partial X^{i}}{\partial x^{i}}-v^{i} \frac{\partial X^{0}}{\partial x^{i}}-\Gamma\left(X^{0}\right)\right)
$$

which can be rewritten as

$$
\operatorname{div}\left(X^{(1)}\right)=2 \sum_{i=1}^{n}\left(\frac{\partial X^{i}}{\partial x^{i}}-v^{i} \frac{\partial X^{0}}{\partial x^{i}}\right)-(n-1) \Gamma\left(X^{0}\right)
$$

and, as det $W$ is a Jacobi multiplier, the constant of the motion corresponding to (4.3) is (5.15), in agreement with the above mentioned results in [22].

\section{Conclusions and outlook}

It has been shown how the use of basic results of the differential geometry concerning the concept of divergence of a vector field, as well as some properties relating Lie 
derivatives of forms with respect to couples of vector fields, allows us to establish in Theorem 1 a method to find a first-integral for a divergence-free vector field when a symmetry vector field is known. The theory is generalised to include a more general vector field when a Jacobi multiplier for such given vector field is known in Theorem 2. Theorem 3 presents the extension of the results of these Theorems when a normalizer vector field instead of a symmetry vector is known. This generalization is very relevant for the case of a nonautonomous system. The main interest of these Theorems is that they present in an intrinsic way a general result that contains as particular cases many expressions of first-integrals or constants of motion derived by ad-hoc methods in many branches of physics. This makes very interesting this unifying way of finding them using geometric tools of modern differential geometry. Several examples of applications to general Hamiltonian systems, second order differential equations and Lagrangian systems, both in the autonomous and the nonautonomous cases, have also been exhibited.

\section{References}

[1] D.G. Currie and E.J. Saletan, q-equivalent particle Hamiltonians. The classical one-dimensional case, J. Math. Phys. 7, 967-974 (1966).

[2] S. Hojman and H. Harleston, Equivalent Lagrangians: multidimensional case, J. Math. Phys. 22, 1414-1419 (1981).

[3] J.F. Cariñena and L.A. Ibort, Non-Noether constants of motion, J. Phys. A: Math. Gen. 16, 1-7 (1983).

[4] S. Hojman, A new conservation law constructed without using either Lagrangians or Hamiltonians, J. Phys. A. Math. Gen. 25, L291-L295 (1992).

[5] F. González-Gascón, Geometric foundations of a new conservation law discovered by Hojman, J. Phys. A: Math. Gen. 27, L 59-L 60 (1994).

[6] F. Darabi, M. Golmohammadi, and A. Rezaei-Aghdam, FRW string cosmological solutions via Hojman symmetry, Int. J. Geom. Methods Mod. Phys. 17, 2050175 (2020).

[7] F. Darabi, M. Golmohammadi, and A. Rezaei-Aghdam, Generalized (2 + 1)-dimensional BTZ black holes via Hojman symmetry, arXiv:2010.08424v2 (2021). 
[8] H. Wei, Y-N. Zhou, H-Y. Lii and X-B. Zou, Hojman symmetry in $f(T)$ theory, Astrophys. Space Sci. 360, 6 (2015).

[9] H. Wei, Y-N. Zhou, H-Y. Lii and X-B. Zou, Exact cosmological solutions of $f(R)$ theories via Hojman symmetry, Nucl. Phys. B 903, 132-149 (2016).

[10] S. Capozziello and M. Roshanc, Exact cosmological solutions from Hojman conservation quantities, Phy. Lett. B 726, 471-480 (2013).

[11] M.C. Paolella and S. Capozziello, Hojman symmetry approach for scalartensor cosmology, Phy. Lett. A 379, 1304-1308 (2015).

[12] A. Paliathanasis, P.G.L. Leach and S. Capozziello, On the Hojman conservation quantities in Cosmology, Phy. Lett. B 755, 8-12 (2016).

[13] F. González-Gascón, Notes on the Connection between the Symmetries and the First Integrals of Dynamical Systems, Lett. Nuovo Cim. 19, 366-368 (1977).

[14] M. Lutzky, Remarks on a recent theorem about conserved quantities, J. Phys. A: Math. Gen. 28, L 637-L 638 (1995).

[15] J.F. Cariñena and P. Santos, Jacobi Multipliers and Hamel's formalism, J. Phys. A: Math. Theor. 54, 225203 (2021).

[16] J.F. Cariñena, A. Ibort, G. Marmo and G. Morandi, Geometry from Dynamics: Classical and Quantum. Springer, Dordrecht, 2015.

[17] F. Mei, Non-Noether conserved quantity for differential equations of motion in the phase space, Chin, Sci. Bull. 47, 2049-2050 (2002).

[18] S-L. Gu and K-X, Wei, Study on The Symmetry and Conserved Quantities for Hamilton Systems, International Conference on Logistics Engineering, Management and Computer Science (LEMCS 2014), Advances in Intelligent Systems Research Series, p. 798-801 (2014).

[19] M. Crampin, Constants of the Motion in Lagrangian Mechanics, Int. J. Theor. Phys. 16, 741-754 (1977).

[20] J.F. Cariñena, J. Fernández-Núñez and E. Martínez, A geometric approach to Noether's Second Theorem in time-dependent Lagrangian Mechanics Lett. Math. Phys. 23, 51-63 (1991). 
[21] J.F. Cariñena, J. Fernández-Núñez and E. Martínez, Noether's theorem in time-dependent Lagrangian Mechanics, Rep. Math. Phys. 31, 189-203 (1992).

[22] H-B. Zhang and L-Q. Chen, The Unified Form of Hojman's Conservation Law and Lutzky's Conservation Law, J. Phys. Soc. Japan 74, 905-909 (2005).

[23] F. González-Gascón, Divergence-free vector fields and integration via quadratures, Phys. Lett. A 225, 269-273 (1996).

[24] M. Lutzky, Conserved quantities and velocity-dependent symmetries in Lagrangian dynamics, Int. J. Nonlinear Mechanics 33, 393-396 (1998).

[25] W.-L. Huang and J.-L. Cai, Inverse Problems of Mei Symmetry for Nonholonomic Systems with Variable Mass, J. Mech. 31, 515-523 (2015).

[26] S. Hojman, Symmetries of Lagrangians and of their equations of motion, J. Phys. A, Math. Gen. 17, 2399-2412. (1984).

[27] S. Hojman, Interpretation of symmetry transformations, J. Phys. A, Math. Gen. 17, L521-L525 (1984).

[28] S-K. Luo, New Types of the Lie Symmetries and Conserved Quantities for a Relativistic Hamiltonian System, Chinese Phys. Lett. 20, 597-599 (2003).

[29] Y.-L. Han, X.-X. Wang, M.-L. Zhang and L.-Q. Jia, Special Lie symmetry and Hojman conserved quantity of Appell equations for a Chetaev nonholonomic system, Nonlinear Dyn. 73, 357-361 (2013).

[30] Y.-L. Han, X.-X. Wang, M.-L. Zhang and L.-Q. Jia, Lie Symmetry and Approximate Hojman Conserved Quantity of Lagrange Equatio ns for a Weakly Nonholonomic System, J. Mech. 30, 21-27 (2014).

[31] J-L. Fu, L-Q. Chen, S. Jiménez and Y-F. Tang, Non-Noether symmetries and Lutzky conserved quantities for mechanico-electrical systems, Phys. Lett. A 358, 5-10 (2006). 